\title{
Modulation Coding Scheme Performance Analysis (Mcs) Wireless 802.11ac Indoor At 5 Ghz Frequency In West And East Telecommunication Labs Politechnic State Of Semarang
}

\author{
Dimas Tampan Setiadi ${ }^{1}$, Subuh Pramono. ${ }^{2}$, Budi Basuki ${ }^{3}$ \\ 1,2,3 Majors Technic Electro, Politeknik Negeri Semarang \\ dimassetiadi081294@gmail.com ${ }^{1}$, subuhpramono@polines.ac.id ${ }^{2}$, budi.basuki2010@gmail.com ${ }^{3}$
}

\begin{abstract}
Modulation Coding Scheme or MCS is a value that specifies the modulation, coding and number of spatial streams and produces a fixed rate data rate based on channel conditions and RSSI values received by the user. Modulation on MCS there are 5 kinds of BPSK, QPSK, 16 QAM, 64 QAM and 256 QAM. While the coding rate there are 4 kinds of $1 / 2,2 / 3,3 / 4$ and $5 / 6$. MCS and RSSI. Changes can not be separated from wireless propagation that includes Large Scale Propagation and Small Scale Fading in particular the existence of obstacles in the form of objects and indoor or outdoor areas. Test results show that major changes to RSSI are influenced by indoor or outdoor areas. MCS changes are uncertain and not RSSI based. MCS and RSSI will change based on channel conditions.
\end{abstract}

Index Terms - MCS, RSSI, indoor, outdoor

\section{PRELIMINARY}

The development of internet with wireless technology is growing. Increasing number of internet users then it takes also a large and stable data transfer. But to get stable data depends on the condition of the existing environment. The propagation condition of the ideal wireless channel is LOS (Line Of Sight) but in reality the line between the transmitter and receiver is almost never LOS. That is why this wireless propagation process of reflection, diffraction and scattering mechanism. Because these processes the received signal to the receiver is a combination of signals with different amplitude and phase delay. In other words, the transmitted signal fluctuates due to the fluctuating channel characteristics. Fluctuations are divided into two things: Large Scale Propagation and Small Scale Fading (Multipath).

In Small Scale Fading an amplitude fluctuation occurs due to destructive or constructive superposition (Time Variant Channel) and time dispersion (Time Spreading) of the received signal due to different arrival times of different paths (Multipath).

Modulation Coding Scheme (MCS) includes modulation and coding rate. Modulation on MCS there are 5 types of BPSK, QPSK, 16QAM, 64QAM, 256QAM. The constellation diagram for 64QAM modulation has 64 symbols, each symbol consisting of 6 binary bits.

While the coding rate on Modulation Coding Scheme (MCS) consists of error detection and bandwidth efficiency. The BPSK modulation has a coding rate of $1 / 2$. This indicates the existence of 1 bit data information after dikodingkan results output into 2 bits of information data. The bandwidth efficiency at the coding rate is $50 \%$, because the data required is only 1 bit of information (half of the data bits overall).

\section{THEORICAL BASIS}

\section{A. Wireless Communication}

Wireless communication is very fast in its development in the communications industry. It gets a lot of attention from the media and from the public. Local wireless networks are now replacing cabling systems at home, business and universities. Many applications, including wireless networks, automated factories, smart homes and medical devices. However, many challenges are technically in making wireless network designs that deliver the performance needed to support applications [1].

\section{B. Radio Wave Propagation}

Electromagnetic waves propagate through environments in which they are reflected, scattering and 
diffraction against walls, buildings and so on. To know this usually use the ray-tracing technique. This technique is used in electromagnetic propagation by displaying waves as particles [1].

\section{Modulation Coding Scheme (MCS)}

The selection of modulation and coding on MCS is much easier than the previous generation of $802.11 \mathrm{n}$ which has 70 more while in 802.11 ac it only has 10 . Modulation explains how many bits are there during transmission. The higher the modulation the more data when transmitted, but it requires a higher SNR. The principal error-correcting code is to add information according to the size shown code rate. Code rate $R=1 / 2$ sends 1 bit of data for every 2 bits. The higher the code rate has more data and the lower correction bits, so it can not handle many errors. In 802.11ac, modulation and coding rate converge into single numbers, the MCS Index. Each MCS value can ensure data rate speed but it depends on channel width, number of spatial streams and guard intervals [2].

\section{Modulasi Digital}

Requirement to send digital information in bits using digital modulation from analog modulation. Generally digital modulation techniques require analog signal messages converted into bits, then convert bits into baseband digital signals, which can be used to modulate the carrier to create digitally modulated signals. Moreover, bits can be shown with different types of baseband digital signals to form a modulated signal spectrum. Bit can also be encoded on the transmitter and encoded on the receiver to correct errors due to noise and interference. Transmission security also includes the features of digital modulation. It would be easier to protect the message signal on the carrier with a modulated digital signal [2].

\section{E. Coding Rate}

Of the many applications, coding rates are always represented by fractions of the total channel capacity, the remainder being used as forward error correction (FEC). This coding scheme is made to tolerate received errors in overall performance, for example signal quality. By leveraging its durability, lower FECs can be used and higher coding capacity can provide better data quality [3].

\section{F. Receive Signal Strength Indicator (RSSI)}

RSSI is a technology used to measure the signal strength indicators received by a wireless device. However, direct mapping of the distance-based RSSI values has many limitations, since RSSI is essentially vulnerable to noise, multi-path fading, interference, and so on which results in large fluctuations in received power [4].

\section{SYSTEM DESIGN}

\section{A. System Design}

The network system built is a WLAN network using the IEEE 802.11ac standard. The Access Point placed in the room will result in the scope of the Modulation Coding Scheme (MCS) that generates the data rate for each user in different positions as in Figure 1.

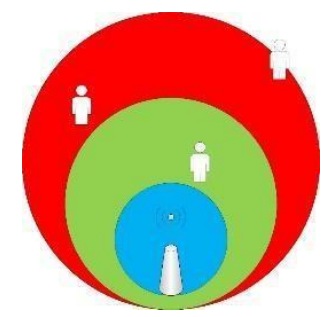

Figure 1. Overview of MCS coverage

\section{B. Access Point Installation}

Installation of access point (AP) in Eastern Lab is done at 3 positions near the door of East Lab 2 which can be seen in Figure 2, then on the east side as in Figure 3 and the third installation is on the south side as in Figure 4.

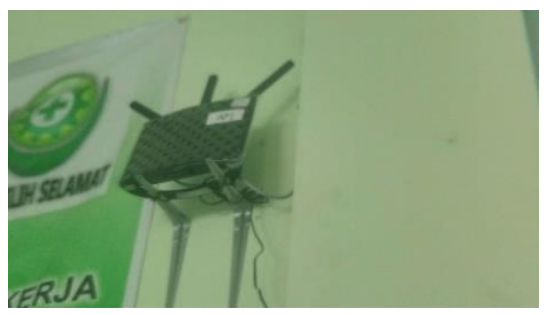

Figure 2. The first AP position in East Lab 2

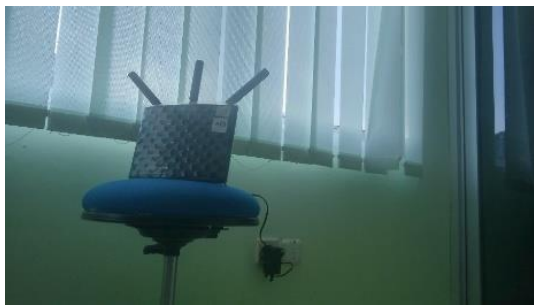

Figure 3. The second AP position in East Lab 2 


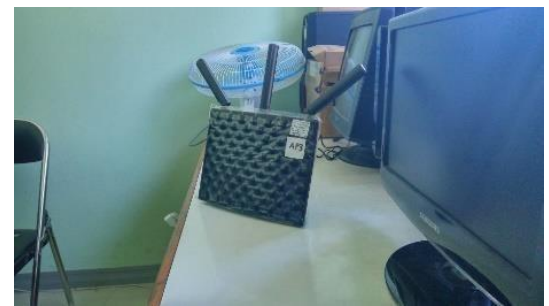

Figure 4. The third AP position in East Lab 2

Then for the installation of access point in Lab 1 also there are 3 positions on the south side as in Figure 5. The second is on the west side as in Figure 6. And the third is on the north end of the room as shown in Figure 7.

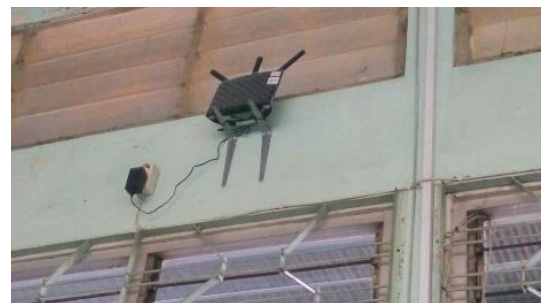

Figure 5. The first AP position in East Lab 2

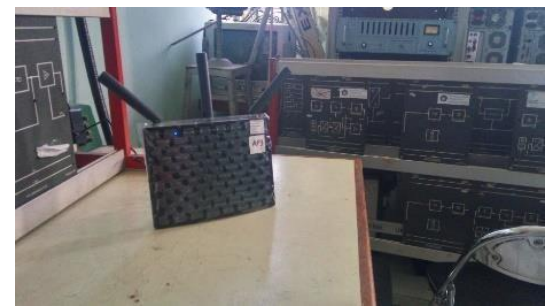

Figure 6. The second AP position in East Lab 2

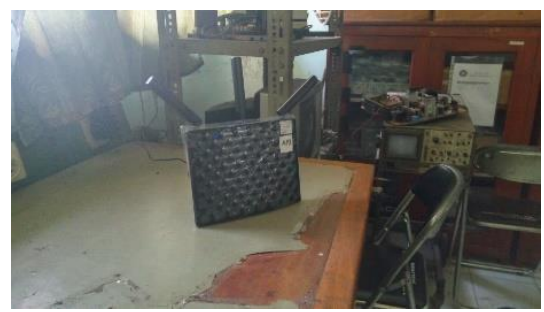

Figure 7. The third AP position in East Lab 2

\section{Transmitter Design}

The design of the transmitter is done in Lab East 2 and West Lab 1 by installing access points on 3 different places in turn.

The first transmitter is placed in East Lab 2 as in Figure 8. Then the second transmitter is placed in East Lab 2 as in Figure 9 and the third transmitter is placed in East Lab 2 as in Figure 10.
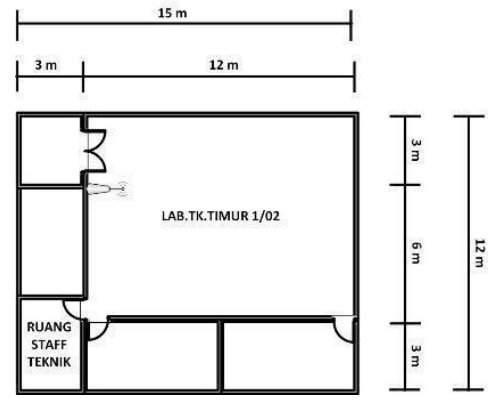

Gambar 8. The first transmitter position in East Lab 2

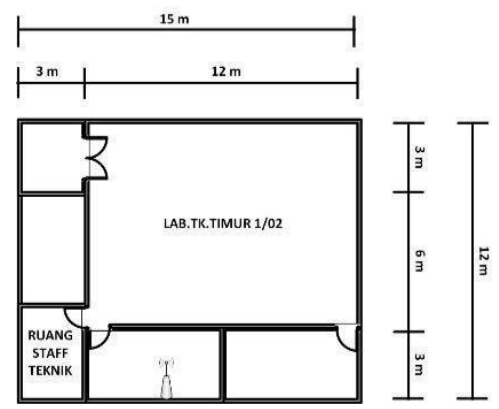

Gambar 9. The second transmitter position in East Lab

2
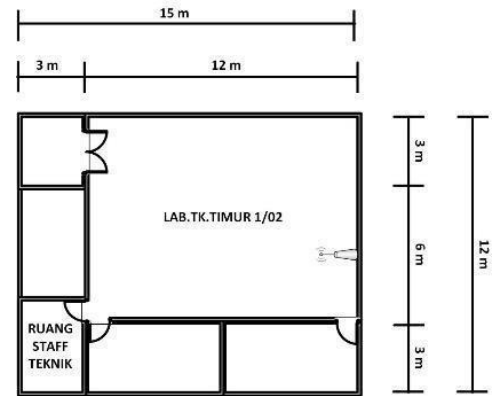

Gambar 10. The third transmitter position in East

Lab 2

Then in the first West Lab Transmitter is placed in the West Lab 1 as in Figure 11. Then the second transmitter is placed in the West Lab 1 as in Figure 12 and the third transmitter is placed in the West Lab 1 as in Figure 13.

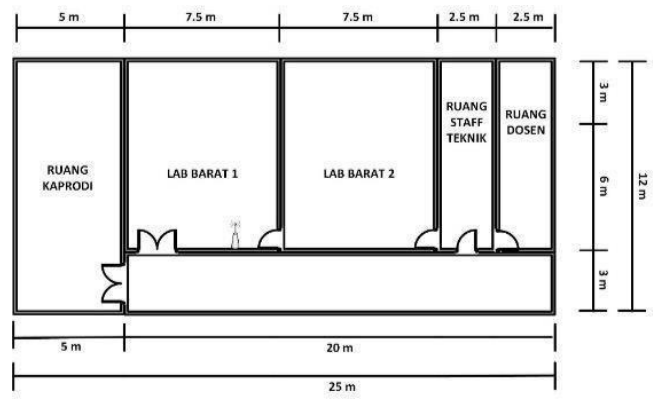

Figure 11. The first transmitter in the West Lab 1 


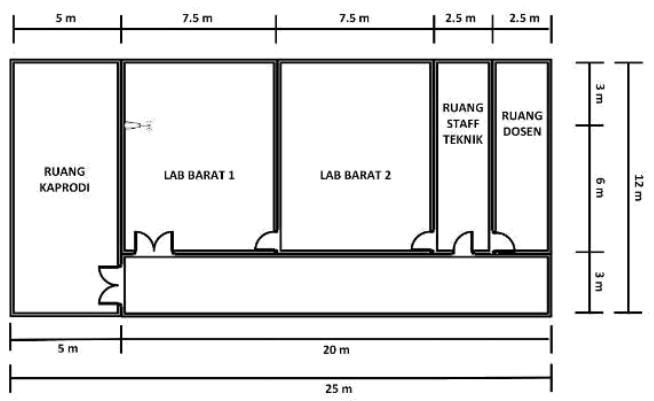

Figure 12. The second transmitter in the West Lab 1

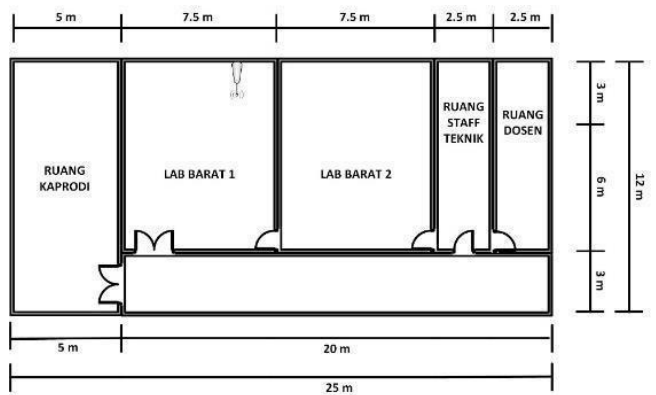

Figure 13. The third transmitter in the West Lab 1

\section{TESTING METHODS}

The method used is path test. The test is divided into 2 parts, namely the test path in east lab 2 and west lab 1 . Each room there are 3 test lines with different path conditions. That way the value of MCS and RSSI will be known by the existence of the test track.

\section{RESULT AND DISCUSSION}

The result of the test path in Lab East 2 is divided into 2 which are RSSI and MCS. Test results are generated from each path. In line 1 can be seen in Figure 14. The results of path 2 testing can be seen in Figure 15 and the results of path 3 testing can be seen in Figure 16.

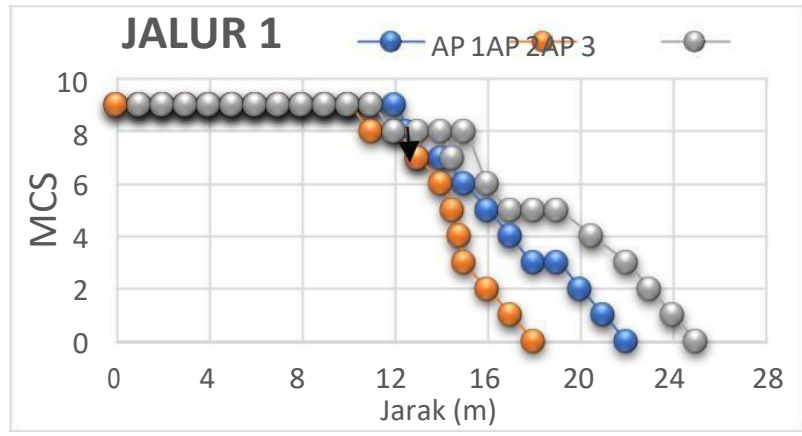

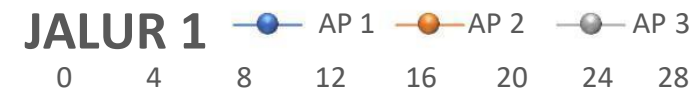

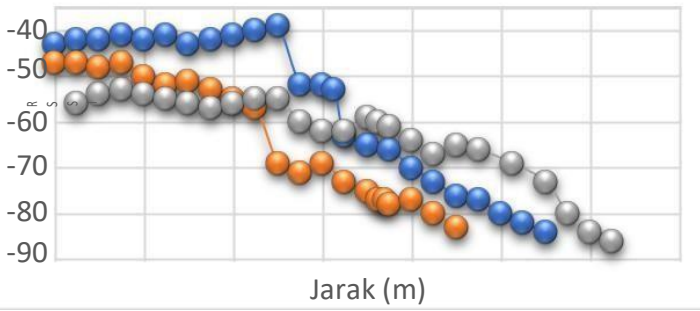

Figure 14. Results of MCS and RSSI lane 1 East Lab 2

When viewed on the value of both MCs AP has the same value, especially when the user is in the room. The value of MCS 9 obtained by the user due to the condition of the Line Of Sight (LOS). Differences in MCS values can be seen when the user is outside the room on the 13th meter. When MCS closed door condition is obtained is MCS 7 while in MCS open door condition obtained is MCS 8. It relates to door barrier that is in East Lab 2 so it influences with MCS value obtained by user. Even because of the resistance, the minimum value of MCS on line 1 (closed door) can only reach at 22 meters while on the 2 nd line (open door) the minimum value of MCS can reach 24 meters. Door barriers in Eastern Lab 2 not only affect the value of MCS but also the value of RSSI, especially when the user is outdoors that is at the 11th meter. The drastically different $\mathrm{dBm}$ difference exists at the 10th meter to the 11th meter with a value of -39 $\mathrm{dBm}$ to $-52 \mathrm{dBm}$ for closed door conditions. As for the open door condition has $\mathrm{dBm}-41 \mathrm{dBm}$ to $-49 \mathrm{dBm}$.

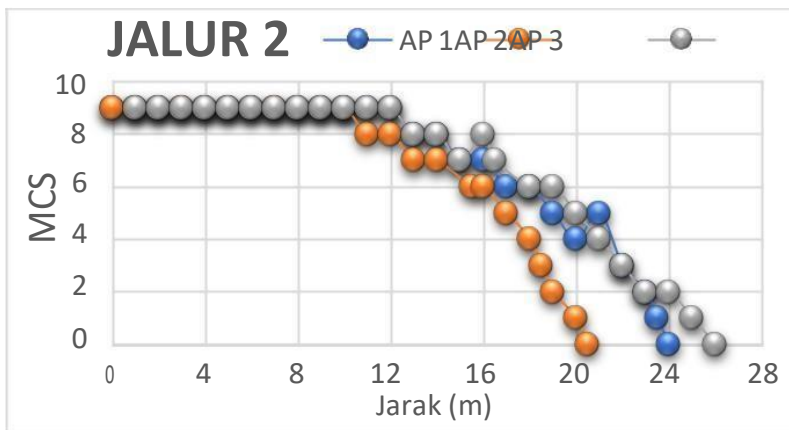




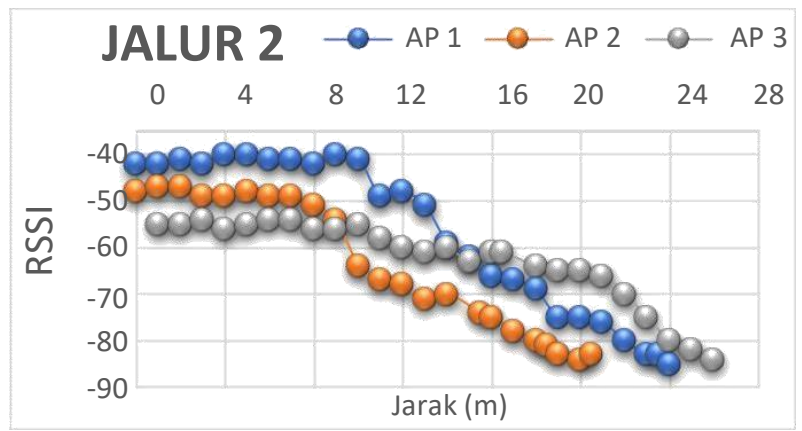

Figure 15. Results of MCS and RSSI lane 2 East Lab 2

It can be seen at the 10th meter that the MCS 9 value is only obtained by the user when indoors. When the user is outdoors, MCS turns into MCS 8 even if the room is closed or opened so that the door barrier is equally affecting the value of MCS. However, due to closed door barriers, MCS values may be lower than MCS values when the door is opened ie MCS 0 when at the 18th meter (closed door) and MCS 0 at 20.5 meters (open door).

When viewed from the three access point positions have an equivalent MCS value between the same path, the path 1 and line 2. However, the best RSSI value generated access point with the first position because the position has a value of $-42 \mathrm{dBm}$ when the user is in the room and when user is outside the room has a value of $49 \mathrm{dBm}$. Then in that position also has the best MCS value that is MCS 9 on the 12th meter with the value of RSSI $-52 \mathrm{dBm}$. In the second and third AP positions the maximum value of MCS 9 is obtained only at the 10th and 11th meters. With so the reach of the farthest MCS 9 generated AP with the first position so that for users who are outside the room with the position of the 12th meter can use the internet with the quality of MCS 9. MCS 0 range on the third AP is in the third AP position up to the meter to- 26. However, the third AP is not appropriate if it is a permanent position due to the low RSSI value, so even if the AP coverage is wide but the user will get a weak signal. In addition, obstacles in the form of doors also affect the RSSI though not too big.

On the third test path to the Engineering staff room with 3 different AP positions. Results of MCS and RSSI can be seen in Figure 16.

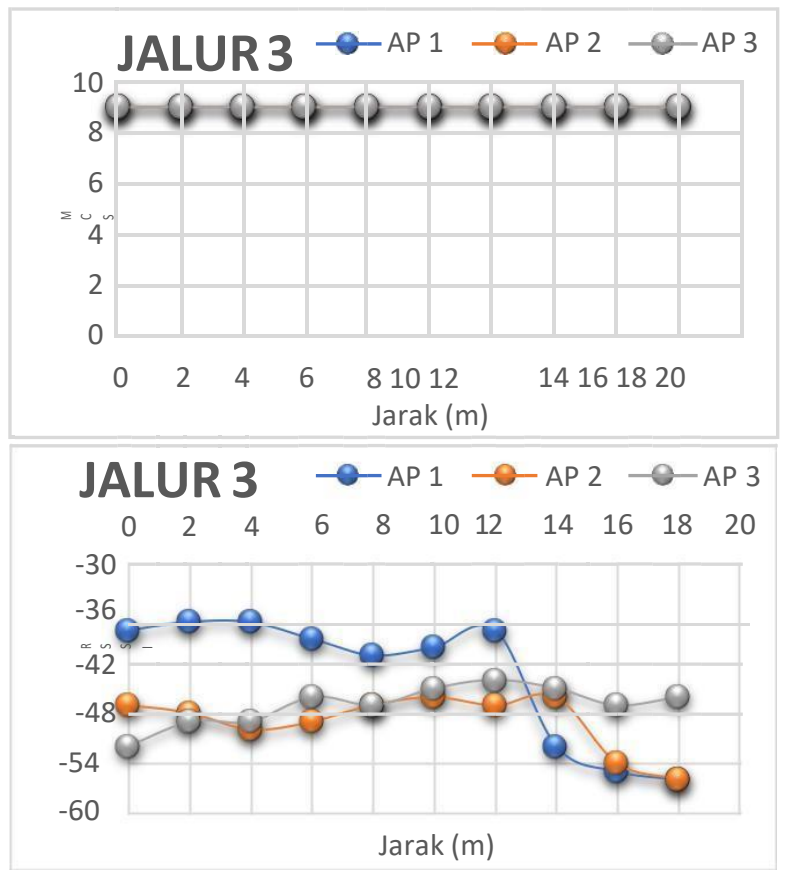

Figure 16. Results of MCS and RSSI lane 3 East Lab 2

MCS AP positions 1, 2 and 3 have the same value that is MCS 9. Nevertheless the position of the right access point can be seen from the other side of the value of RSSI. The RSSI value on all three devices is unstable, especially for the first position RSSI access point value. The first access point has a good RSSI value of $-37 \mathrm{dBm}$, but when the user reaches the 12th meter the RSSI value drops drastically to $-52 \mathrm{dBm}$ to $-56 \mathrm{dBm}$. This will certainly affect the signal strength, especially on users who are in the Technical Staff Room. As with the second access point position, it has a low RSSI value of $-47 \mathrm{dBm}$ to $-56 \mathrm{dBm}$. Unlike the third access point position that has RSSI $-46 \mathrm{dBm}$ value when the user is in the Technical Staff Room even though this third access point position has bad RSSI value when the user is in the middle room of Eastern Lab class 2 because it has the value of RSSI $-52 \mathrm{dBm}$. If taken conclusion then third position access point is the right position for user is in Technical Staff Room. While the first position access point is the right position for the user who is in the middle room of East Lab 2.

Then MCS and RSSI in Lab 1 also have 3 lanes. The results of the first path can be seen in Figure 17. The result of the second line can be seen in Figure 18 and the third line can be seen in Figure 19. 


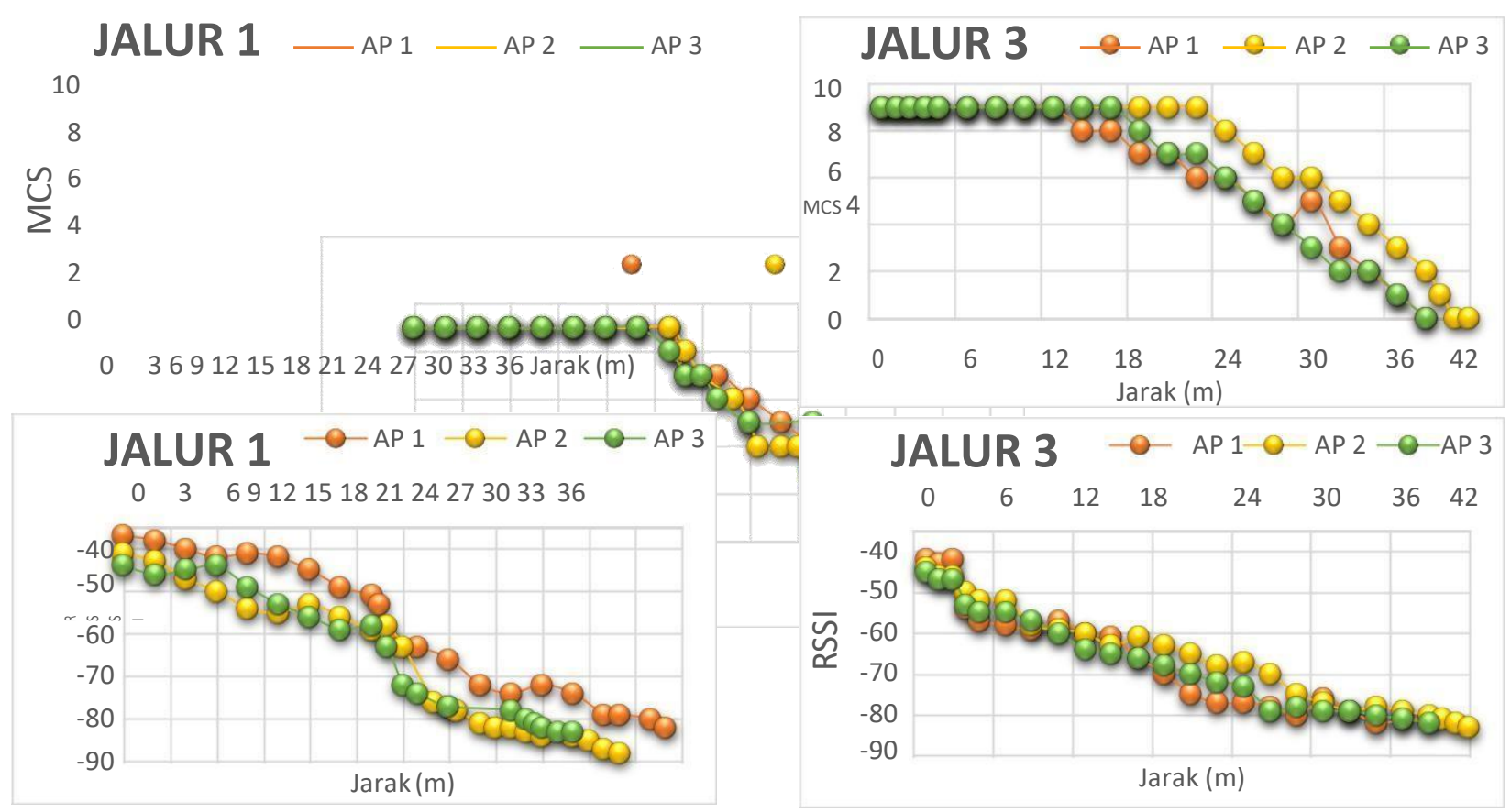

Figure 17. Results of MCS and RSSI lane 1 West Lab 1

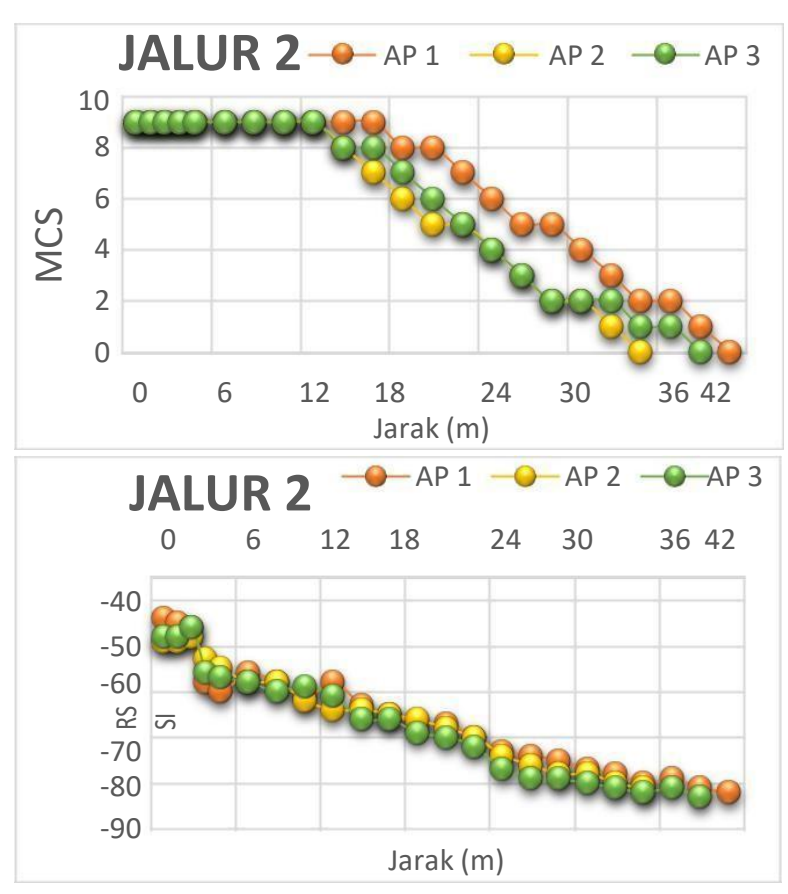

Figure 19. Results of MCS and RSSI lane 3 West Lab 1

With the three access point locations it can be seen that in the first line the value of the farthest MCS 9 is in the position of the first AP and the second is on the 16th meter. But on the first AP has the value of RSSI better than the position of AP 2 with a value of $-37 \mathrm{dBm}$. This will affect the signal quality of the user. While on the second and third lines for the third AP it can be seen that in the outdoor area changes the value of MCS 9 to MCS 8 occurs when the value of RSSI $-60 \mathrm{dBm}$ range. However, the value of RSSI in the first AP is higher than the AP in the second and third position that has a value of $-46 \mathrm{dBm}$ and $-42 \mathrm{dBm}$. However, the MCS value is further in reach of the second access point position that reaches up to 23 meters. However, if considered with the first line, the correct access point position for the West Lab Room 1 is the first position access point due to having a good RSSI signal and MCS coverage which is almost equivalent to the access point position 2. In addition, obstacles are doors and indoor or indoor area. outdoor effect on RSSI. 


\section{CLOSING}

\section{A. Conclusion}

Based on the results of testing and analysis that has been done can be concluded as follows:

1. The best access point position in Eastern Lab 2 is the access point with the first position, because in that position has the best RSSI is -43 $\mathrm{dBm}$ and in that position has the furthest MCS that is at the 12th meter with the value of MCS 9.

2. The change of MCS value to RSSI is so tight that the change of MCS niali can not be ascertained by the value of RSSI.

3. Obstacles in the form of doors and indoor or outdoor areas have an effect on RSSI.

\section{B. Suggestion}

Based on test results and analysis can be given some suggestions as follows:

1. Installation of access points do not corner the room far from the most users.

2. Installation of access points placed close to the indoor and outdoor areas so that users who are in both areas can connect the Internet network with a good RSSI signal.

3. With the latest wireless technology is expected to replace previous wireless devices that are not feasible to use due to low data distribution.

4. Expected utilization AC15 Access Point tent can be developed again for students of Electrical Engineering in the future.

\section{REFFERENCE}

[1] Goldsmith, A. (2005). Wireless Communication. Cambridge.

[2] IEEE. (2009). IEEE Standart for Information Technology-Telecommunication and Iformation Exchange between System-Local Area NetworkSpecific Requirements. New York : IEEE Computer Society.

[3] Kondoz, A. (2004). Digital Speech Coding for Low Bit Rate Communication System. Chicester : Jhon Wiley and Sons.

[4] Sahu, P. W. Dual RSSI Trend Based Localization for Wireless Sensor Network. 